\title{
Plasmons and Screening in Monolayer and Multilayer Black Phosphorus
}

\author{
Tony Low, ${ }^{1,2,5,{ }^{*}}$ Rafael Roldán, ${ }^{3}$ Han Wang, ${ }^{6}$ Fengnian Xia, ${ }^{2}$ Phaedon Avouris, ${ }^{1}$ \\ Luis Martín Moreno, ${ }^{4}$ and Francisco Guinea ${ }^{3}$ \\ ${ }^{1}$ IBM T.J. Watson Research Center, 1101 Kitchawan Road, Yorktown Heights, New York 10598, USA \\ ${ }^{2}$ Department of Electrical Engineering, Yale University, New Haven, Connecticut 06511, USA \\ ${ }^{3}$ Instituto de Ciencia de Materiales de Madrid, CSIC, Sor Juana Inés de la Cruz 3, 28049 Madrid, Spain \\ ${ }^{4}$ Instituto de Ciencia de Materiales de Aragón and Departamento de Física de la Materia Condensada, \\ CSIC-Universidad de Zaragoza, E-50009 Zaragoza, Spain \\ ${ }^{5}$ Department of Electrical and Computer Engineering, University of Minnesota, Minneapolis, Minnesota 55455, USA \\ ${ }^{6}$ Department of Electrical Engineering, University of Southern California, Los Angeles, California 90089, USA
}

(Received 15 April 2014; published 4 September 2014)

\begin{abstract}
Black phosphorus exhibits a high degree of band anisotropy. However, we find that its in-plane static screening remains relatively isotropic for momenta relevant to elastic long-range scattering processes. On the other hand, the collective electronic excitations in the system exhibit a strong anisotropy. Band nonparabolicity, due to interband couplings, leads to a plasmon frequency which scales as $n^{\beta}$, where $n$ is the carrier concentration, and $\beta<\frac{1}{2}$. Screening and charge distribution in the out-of-plane direction are also studied using a nonlinear Thomas-Fermi model.
\end{abstract}

DOI: 10.1103/PhysRevLett.113.106802

PACS numbers: 73.21.-b, 73.22.Lp, 73.61.-r, 74.78.Fk

Introduction.-Black phosphorus (BP) is one of the thermodynamically more stable phases of phosphorus, at ambient temperature and pressure. It is a layered material, with each layer forming a puckered surface due to $s p^{3}$ hybridization. In its bulk crystalline form [1-5], BP is a semiconductor with a direct band gap of about $0.3 \mathrm{eV}$ with measured Hall mobilities in $n$-and $p$-type samples approaching $10^{5} \mathrm{~cm}^{2} / \mathrm{V} \mathrm{s}$, and highly anisotropic electrical and optical properties. Recently, experiments emerged also on its multilayer form [6-13].

In this Letter, we examine the collective electronic excitations of BP, and its electrostatic screening behavior both along the in- and out-of-plane directions. We calculate the dielectric function $\epsilon(\mathbf{q}, \omega)$, at finite frequency $\omega$ and wave vector $\mathbf{q}$, within the random phase approximation (RPA), using an effective low-energy Hamiltonian [14]. The inherent anisotropy of screening is studied, and the out-of-plane screening properties of multilayer BP flakes are considered within a nonlinear Thomas-Fermi model. The 2D plasmon modes show a highly anisotropic dispersion, $\omega_{p l}(\mathbf{q}, \omega)$, and we studied its scaling behavior with doping. Lastly, we discuss the implications of our results for basic electrical and light scattering experiments.

Hamiltonian.-BP has an orthorhombic crystal structure consisting of puckered layers. The lattice constant in the outof-plane direction is about $10.7 \AA$, and the effective layer-tolayer distance is half of this value [4]. In monolayer BP, translational symmetry in the $z$ direction is broken, and its band structure has a direct energy gap at the $\Gamma$ point instead of the $Z$ point in the bulk case. Based on $\mathbf{k} \cdot \mathbf{p}$ theory and symmetry arguments, the in-plane electron dispersion around the $\Gamma$ point can be described by the following low-energy Hamiltonian [14]:

$$
\mathcal{H}=\left(\begin{array}{cc}
E_{c}+\eta_{c} k_{x}^{2}+\nu_{c} k_{y}^{2} & \gamma k_{x}+\beta k_{y}^{2} \\
\gamma k_{x}+\beta k_{y}^{2} & E_{v}-\eta_{v} k_{x}^{2}-\nu_{v} k_{y}^{2}
\end{array}\right),
$$

where $\eta_{c, v}$ and $\nu_{c, v}$ are related to the effective masses, while $\gamma$ and $\beta$ describe the effective couplings between the conduction and valence bands. $E_{c}$ and $E_{v}$ are the energies of the conduction and valence band edges. The energy gap for monolayer BP has not been measured, but recent ab initio calculation based on the $G W$ method found an energy gap of $\sim 1.5-2$ eV $[15,16]$.

Unlike other layered materials such as graphene and the transition metal dichalcogenides (TMDs), electrons in BP are energetically highly dispersive along the out-of-plane direction. Cyclotron resonance experiments on bulk BP [17] found an out-of-plane effective mass considerably smaller than that of TMDs [18]. For multilayer BP, confinement in the out-of-plane $z$ direction leads to multiple subbands. The in-plane dispersion within each subband $j$ can be described by Eq. (1), where $E_{c, v}$ are being replaced with $E_{c, v}^{j}$. More explicitly, $\delta E_{c}^{j}$ is given by $j^{2} \hbar^{2} \pi^{2} / 2 m_{c z} d^{2}+\delta_{c}(d)$, where $j$ labels the subband, $d$ is the thickness of the BP film, and $m_{c z}$ is the electron effective mass along $z$. Analogous expressions apply also for the hole case. The quantities $\delta_{c, v}(d)$ are chosen such that it reproduces the energy gap of the BP film [15], of 2 and $0.3 \mathrm{eV}$ in the monolayer and bulk limit, respectively. In this work, we adopt an average of the experimental [17] and theoretically $[12,17]$ predicted quantization mass, i.e., $m_{c z} \approx 0.2 m_{0}$ and $m_{v z} \approx 0.4 m_{0}$.

The in-plane dispersion is mainly determined by the parameters $\eta_{c, v}, \nu_{c, v}$, and $\gamma$. These parameters are chosen such that they yield the known anisotropic effective masses. 
In the bulk BP limit, we have $m_{c x}=m_{v x}=0.08 m_{0}$, $m_{c y}=0.7 m_{0}$ and $m_{v y}=1.0 m_{0}[4,17]$, and $m_{c x}=m_{v x} \approx$ $0.15 m_{0}$ for monolayer BP [12]. Using this knowledge, we arrive at the following parameter set: $\eta_{c, v}=\hbar^{2} / 0.4 m_{0}$, $\nu_{c}=\hbar^{2} / 1.4 m_{0}, \nu_{v}=\hbar^{2} / 2.0 m_{0}$, and $\gamma=4 a / \pi \mathrm{eVm}$. The value of $\beta$ is taken to be $\approx 2 a^{2} / \pi^{2} \mathrm{eV} / \mathrm{m}^{2}$ [12], where $a \approx$ $2.23 \AA$ and $\pi / a$ is the width of the $\mathrm{BZ}$ in the $x$ direction.

Dielectric function.-The dielectric function of an electron gas in the RPA can be written as

$$
\epsilon(\mathbf{q}, \omega)=\kappa+v_{c}(q) \Pi(\mathbf{q}, \omega),
$$

where $v_{c}(q)=e^{2} / 2 \epsilon_{0} q$ is the 2D Coulomb interaction and $\kappa$ describes the effective dielectric constant of the two halfspaces mediums, which for a common $\mathrm{SiO}_{2}$ substrate and air is $\sim 2.5 . \Pi(\mathbf{q}, \omega)$ is the $2 \mathrm{D}$ polarizability (i.e., the pair bubble diagram) given by

$$
\begin{aligned}
\Pi(\mathbf{q}, \omega)= & -\frac{g_{s}}{(2 \pi)^{2}} \sum_{s s^{\prime} j j^{\prime}} \int d \mathbf{k} \frac{f_{s j \mathbf{k}}-f_{s^{\prime} j^{\prime} \mathbf{k}^{\prime}}}{E_{s j \mathbf{k}}-E_{s^{\prime} j^{\prime} \mathbf{k}^{\prime}}+\hbar \omega+i \eta} \\
& \times\left|\left\langle\Phi_{s j \mathbf{k}} \mid \Phi_{s^{\prime} j^{\prime} \mathbf{k}^{\prime}}\right\rangle\right|^{2},
\end{aligned}
$$

where $\mathbf{k}^{\prime}=\mathbf{k}+\mathbf{q},\left\{s, s^{\prime}\right\}= \pm 1$ denote conduction and valence bands, while $\left\{j, j^{\prime}\right\}$ are the subband indices and $g_{s}=2$ is the spin degeneracy. $E_{s j \mathbf{k}}$ and $\Phi_{s j \mathbf{k}}$ are the eigenenergies and eigenfunctions after diagonalizing $\mathcal{H}$. $f_{s j \mathbf{k}}=\left\{\exp \left[\left(E_{s j \mathbf{k}}-\mu\right) / k_{B} T\right]+1\right\}^{-1}$ is the Fermi distribution function, where $\mu$ is the chemical potential. Finite damping can be modeled with the phenomenological broadening term $\eta$. Allowed optical transitions between these quantized subbands occur when $s s^{\prime}= \pm 1$ (i.e., intraand interband processes) and $j=j^{\prime}$. Otherwise the matrix element $\langle\ldots\rangle$ in Eq. (3) vanishes.

Screening in monolayer.-In the static limit, we generalize the well-known analytical form of the polarizability for 2D electron gas (2DEG) [19] to include anisotropy. Since $\omega=0$, we deal only with intraband processes. In the $T \rightarrow 0$ and $\eta \rightarrow 0$ limits, we have [20]

$$
\begin{aligned}
\Pi(\mathbf{q}) & =-\frac{g_{s}}{(2 \pi)^{2}} \int_{0}^{\mathbf{k}_{F}} d \mathbf{k}\left[\frac{1}{E_{\mathbf{k}+\mathbf{q}}-E_{\mathbf{k}}}-\frac{1}{E_{\mathbf{k}}-E_{\mathbf{k}-\mathbf{q}}}\right] \\
& =-\frac{g_{s} m_{d}}{\pi^{2} \hbar^{2}} \int_{0}^{p_{F}} d p \int_{0}^{2 \pi} d \theta \frac{p}{s^{2}-4 p^{2} \cos ^{2} \theta},
\end{aligned}
$$

where we have made the transformation [21],

$$
\mathbf{k} \rightarrow \frac{1}{\sqrt{m_{d}}} M^{(1 / 2)} \mathbf{p} \quad \text { and } \quad \mathbf{q} \rightarrow \frac{1}{\sqrt{m_{d}}} M^{(1 / 2)} \mathbf{s},
$$

where $M$ is the mass tensor with diagonal elements $m_{x}$ and $m_{y}, m_{d}$ is the 2D density-of-states mass given by $\sqrt{m_{x} m_{y}}$, and $p_{F}=\sqrt{2 m_{d} \mu} / \hbar$. After some algebra, we arrive at

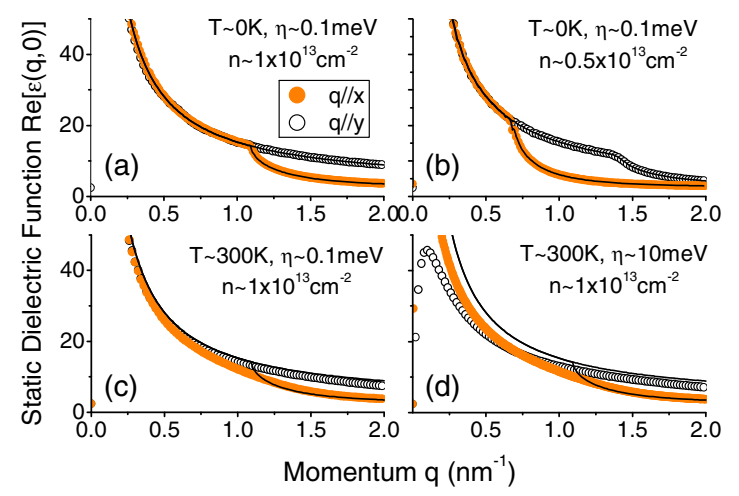

FIG. 1 (color online). Static screening. (a)-(d) Dielectric function for monolayer BP in the static limit, for different conditions as stated. Solid black lines are an analytical expression for $\epsilon(q, 0)$ at $T \rightarrow 0 \mathrm{~K}$ and $\eta \rightarrow 0 \mathrm{eV}$.

$$
\Pi(\mathbf{q})=g_{2 \mathrm{D}} \Re\left[1-\sqrt{1-\frac{8 \mu / \hbar^{2}}{q_{x}^{2} / m_{x}+q_{y}^{2} / m_{y}}}\right]
$$

where $g_{2 \mathrm{D}}=m_{d} / \pi \hbar^{2}$ is the $2 \mathrm{D}$ density of states. We make an interesting remark: for $q \leq 2\left|\mathbf{k}_{F} \cdot \hat{\mathbf{q}}\right|$, we see that $\Pi(\mathbf{q})$ reduces to the familiar relation for the static polarization of a $2 \mathrm{DEG}, \Pi(\mathbf{q})=g_{2 \mathrm{D}}$. Long-range potentials, such as those induced by charged impurities, involve momenta $q$ such that $q \leq 2\left|\mathbf{k}_{F} \cdot \hat{\mathbf{q}}\right|$, so that screening will be isotropic, at least in the zero temperature and disorder limits.

Figure 1(a) compares the static dielectric function obtained numerically with the analytical model in Eq. (6), with excellent agreement in the limits of the model. $\Pi(\mathbf{q})$ has a kink at $q=2\left|\mathbf{k}_{F} \cdot \hat{\mathbf{q}}\right|$. Figure 1 (b) illustrates how the kink migrates with a change in doping. With increasing temperature and disorder, the kink is smoothed out as illustrated in Figs. 1(c)-1(d), showing obvious deviation from the analytical model. The otherwise isotropic screening at small momenta now becomes anisotropic. On the other hand, dynamical screening $\epsilon(\mathbf{q}, \omega)$ in BP exhibits strong directional dependence with $\mathbf{q}$ (see the Supplemental Material [22]).

Plasmon and energy loss function.-The zeros of the dynamical dielectric function $\epsilon(\mathbf{q}, \omega)$ yield the excitation spectrum of the plasmon modes of the electron gas. The

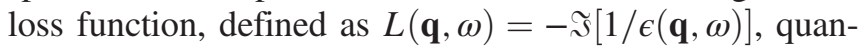
tifies the spectral weight of the plasmon mode, which presents itself as a delta peak in the limit of zero damping. In the long wavelength limit, i.e., $q \ll k_{F}$, these modes are well described by classical Maxwell theory. We consider a BP film sandwiched between two dielectric media, $\epsilon_{1}$ and $\epsilon_{2}$. The bound modes, i.e., plasmons, are characterized by an in-plane wave vector $\mathbf{q}$ pointing at an angle $\theta$ with respect to $x$. The dispersion relation for the bound mode can be obtained from the solution to the following equation:

$$
\left(\bar{Y}_{s}+M_{s s}\right)\left(\bar{Y}_{p}+M_{p p}\right)-M_{p s} M_{s p}=0,
$$


where $\bar{Y}_{\beta}=Y_{\beta}^{1}+Y_{\beta}^{2}(\beta=s, p)$ is the total admittance, with $Y_{s}^{i}=Y_{0}\left(k_{z i} / k_{0}\right)$ and $Y_{p}^{i}=Y_{0} \epsilon_{i}\left(k_{0} / k_{z i}\right)$, and $k_{z i}^{2}=k_{0}^{2} \epsilon_{i}-q^{2}$, $k_{0}=\omega / c . c$ and $Y_{0}=\sqrt{\epsilon_{0} / \mu_{0}}$ are the speed of light and admittance of free space, respectively. The matrix elements of $M$ are expressed in terms of $\sigma_{j j}$, the diagonal components of the 2D BP conductivity tensor,

$$
\begin{aligned}
& M_{s s}=\sigma_{x x} \sin ^{2} \theta+\sigma_{y y} \cos ^{2} \theta, \\
& M_{s p}=M_{p s}=\left(\sigma_{y y}-\sigma_{x x}\right) \sin \theta \cos \theta, \\
& M_{p p}=\sigma_{x x} \cos ^{2} \theta+\sigma_{y y} \sin ^{2} \theta .
\end{aligned}
$$

In the limits $\theta=0, \pi$ and $\sigma_{x x}=\sigma_{y y}$, Eq. (7) reduces to

$$
\bar{Y}_{p}+M_{p p}=0 .
$$

In the nonretarded regime, i.e., $q \gg k_{0}$, hence $k_{z i} \approx i q$, we obtain the "quasistatic" approximation,

$$
-\frac{\sigma_{x x} \cos ^{2} \theta+\sigma_{y y} \sin ^{2} \theta}{\epsilon_{0} \omega}=\frac{\epsilon_{1}}{k_{z 1}}+\frac{\epsilon_{2}}{k_{z 2}} \approx \frac{2 \kappa}{i q},
$$

where $\kappa=\left(\epsilon_{1}+\epsilon_{2}\right) / 2$. For frequencies up to the midinfrared, the conductivity can be approximated by the Drude model,

$$
\sigma_{j j}(\omega)=\frac{i \mathcal{D}_{j}}{\pi(\omega+i \eta / \hbar)}, \quad \mathcal{D}_{j}=\pi e^{2} \sum_{i} \frac{n_{i}}{m_{j}^{i}},
$$

where $\mathcal{D}_{j}$ is the Drude weight and $i$ denotes the subbands. Within the model Hamiltonian, the in-plane electron effective masses in vicinity to the $\Gamma$ point are given by the following expressions [14]:

$$
m_{c x}^{i}=\frac{\hbar^{2}}{2 \gamma^{2} / \Delta^{i}+\eta_{c}}, \quad m_{c y}=\frac{\hbar^{2}}{2 \nu_{c}},
$$

where $\Delta^{i}$ is the subband energy gap. Similar expressions apply for the hole case. Note that in graphene, $\mathcal{D}=\mu e^{2} / \hbar^{2}$ instead. With Eqs. (10) and (11), we have the classical plasmon dispersion along the $j=x, y$ directions, which is $\omega_{p l, j}(\mathbf{q})=\sqrt{\left(\mathcal{D}_{j} / 2 \pi \epsilon_{0} \kappa\right) q}$.

Plasmon in monolayer.-Figure 2(a) plots the RPA loss function $L(\mathbf{q}, \omega)$ for momentum along the two main crystallographic directions for monolayer $\mathrm{BP}$, with an electron doping of $10^{13} \mathrm{~cm}^{-2}$ and $T=300 \mathrm{~K}$. The plasmon disperses differently due to their mass anisotropy, where the smaller mass along $x$ leads to higher resonance frequency. Classical plasmon dispersion agrees well with the RPA result in the long wavelength limit. Because of the energy gap of $2 \mathrm{eV}$ for monolayer BP, Landau damping occurs preferentially via intraband processes. This occurs when the plasmon enters the SP phase space, whose boundaries are given by $\hbar \omega_{\mathrm{SP}}^{ \pm}(\mathbf{q})=E\left( \pm \mathbf{k}_{F}+\mathbf{q}\right)-E\left(\mathbf{k}_{F}\right)$. Our

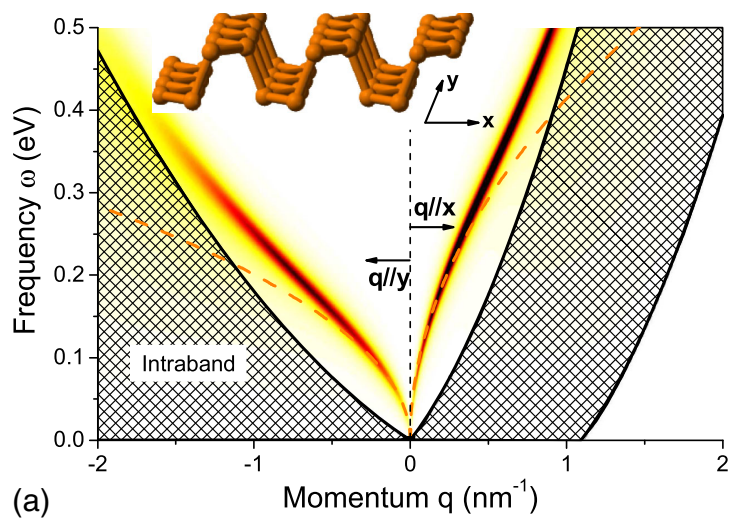

(b)

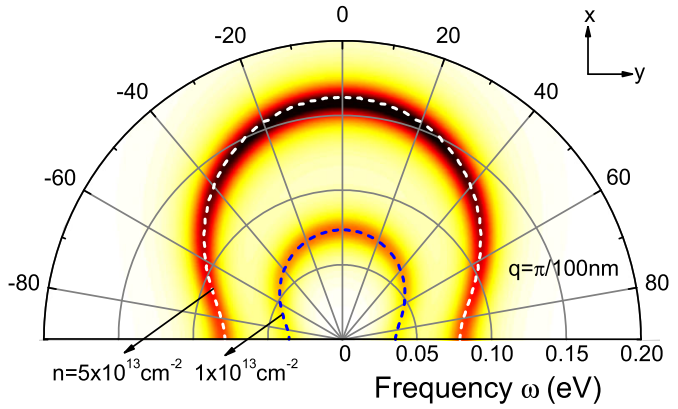

FIG. 2 (color online). Energy loss and plasmon dispersion. (a) Loss function, $L(\mathbf{q}, \omega)=-\Im[1 / \epsilon(\mathbf{q}, \omega)]$, calculated for monolayer BP for electron doping of $1 \times 10^{13} \mathrm{~cm}^{-2}$, for $q$ along the two crystal axes $x$ (right) and $y$ (left). We assumed $T=300 \mathrm{~K}$ and $\eta=10 \mathrm{meV}$. Shaded regions are the Landau damping regions, defined by the single particle phase space $\hbar \omega_{\mathrm{SP}}^{ \pm}(\mathbf{q})$ as described in text. (b) Polar intensity plot of $L(\mathbf{q}, \omega)$ for electron doping of $1 \times 10^{13} \mathrm{~cm}^{-2}$ and $5 \times 10^{13} \mathrm{~cm}^{-2}$ under the same conditions as (a), for specified $q$. The radial and azimuth coordinates denote the frequency $\omega$ and the angular orientation of $q$. Dashed lines are the plasmon solutions from Eq. (7).

calculation suggests that the plasmon along the $y$ direction is damped at midinfrared frequencies, while the plasmon along $x$ persists up to the near infrared. With the assumed $\eta$ in these calculations, the plasmon figure of merit $\mathfrak{R}[q] / \Im[q]$ can approach $\sim 10$.

Plasmon modes in layered materials [23-25] can also be probed by Fourier transform infrared light scattering experiments of nanostructures [26,27] or with infrared nanomicroscopy techniques $[28,29]$. For example, nanostructures exhibit prominent resonances in their extinction spectra due to localized plasmons with an odd multiple of the momentum $q \sim \pi / W$ where $W$ can be the width of nanoribbons, or the diameter of nanodisks. Figure 2(b) shows $L(\mathbf{q}, \omega)$ for a different angular orientation of $\mathbf{q}$ for a momentum corresponding to nanostructures of $100 \mathrm{~nm}$ in size. Dashed lines are solutions of Eq. (7). The results suggest polarization sensitive midinfrared plasmonic resonances in the absorption spectra in BP nanostructures.

Plasmon in multilayers.-Multilayer BP might be more interesting for plasmonics due to the higher carrier 


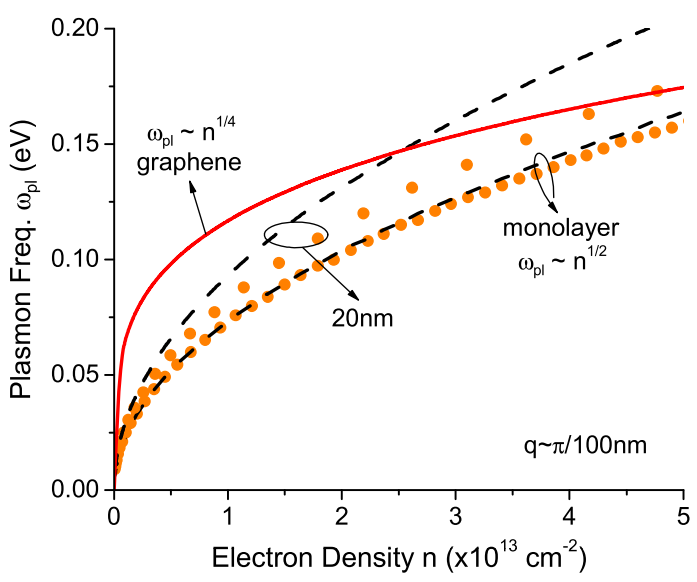

FIG. 3 (color online). Plasmon scaling with carrier concentration. Plasmon energies $\omega_{p l}$, as function of density $n$, calculated for the monolayer and for a $20 \mathrm{~nm}$ BP thick film at a specified $q$ along $x$. Graphene plasmons are shown for comparison. Dashed lines are from Eqs. (10) and (11).

mobilities [7-9]. Figure 3 studies the scaling of plasmon frequency (along $x$ ) with carrier concentration $n$. For monolayer BP, we obtain the expected scaling relation of $\omega_{p l} \propto n^{1 / 2}$, as in conventional 2DEGs. However, for thicker samples, we found that $\omega_{p l} \propto n^{\beta}$, with $\beta<\frac{1}{2}$ instead. This deviation is due to the strong nonparabolicity caused by interband coupling, particularly when the energy gap of the BP film is $\ll \gamma^{2} / \eta_{c, v}$. Hence, nonparabolicity effects are more prominent for thicker films. We also note the general trend of increasing the Drude weight with the film's thickness due to the decreasing effective masses (see the Supplemental Material [22]).

Screening and charge distribution in multilayers.-We complete our study by considering the charge distribution and the electrostatic screening in few-layer BP sheets. For this aim we use a nonlinear Thomas-Fermi theory, which has been shown to properly account for the screening properties of graphite [30-32] and $\mathrm{MoS}_{2}$ [33]. We start by considering a given charge transfer between the substrate and the BP flakes, whose origin can be due to charge impurities in the substrate or to the action of a gate voltage. This charge transfer leads to a net surface-charge density $e n_{0}$ while a layer below the substrate acquires a charge of $-e n_{0}$; see the inset of Fig. 4(a). For a BP sample of thickness $d$, the electrostatic potential $V(z)$ and the carrier distribution $n(z)$ as a function of the distance from the substrate $z$ can be obtained from the energetic balance between kinetic and interlayer capacitance terms, which leads to the nonlinear differential equation [33]

$$
\frac{d^{2} f(z)}{d z^{2}}=\frac{5}{2} \beta_{\perp} f(z)^{3 / 2}
$$

where $f(z)=[e n(z)]^{2 / 3}$ and we have defined $\beta_{\perp}=$ $\left(4 e^{2} / 5 \epsilon_{0} \kappa\right)\left(g_{s} d_{0} m_{d} \sqrt{m_{z}} / 6 \pi^{2} \hbar^{3}\right)^{2 / 3}$, where $d_{0} \approx 1.07 \mathrm{~nm}$ and $\kappa \approx 8.3$ are the interlayer separation and dielectric
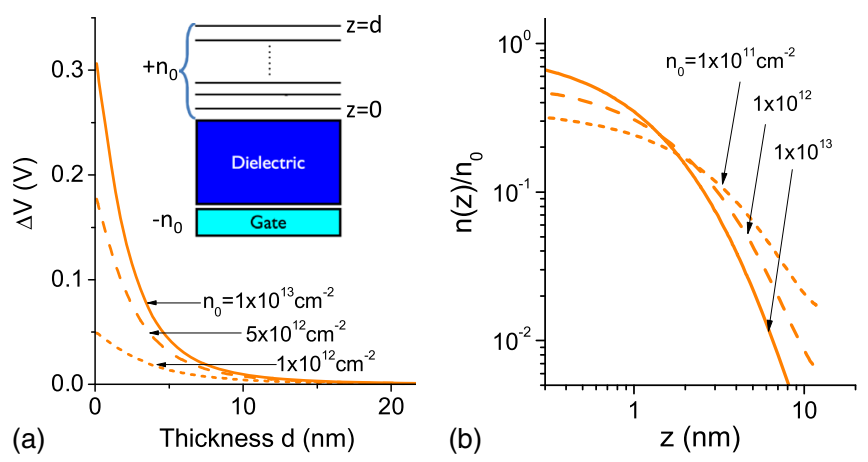

FIG. 4 (color online). Interlayer screening. (a) Potential difference $\Delta V(d)$ as a function of the thickness for an electron doped sample, obtained from the Thomas-Fermi model. Inset shows a sketch of the model. We show the results for different carrier concentrations $n_{0}$. (b) Normalized surface-charge distribution $n(z)$ induced in a $13 \mathrm{~nm}$ thick sample for different gate carrier densities $n_{0}$ as stated.

constant, respectively [4]. Using the boundary conditions $f^{\prime}(0)=\frac{5}{2} \beta_{\perp} e n_{0}$ and $f^{\prime}(d)=0$, one can obtain the charge density from the solution of the integral equation

$$
\int_{f(0)}^{f(z)} \frac{d f}{\sqrt{f^{5 / 2}-f^{5 / 2}(d)}}=\sqrt{\frac{2 \beta_{\perp}}{d_{0}}} z .
$$

On the other hand, the potential difference across a BP sample of thickness $d$ can be shown to be given by [33]

$$
\Delta V(d)=\frac{2 e^{2}}{5 \epsilon_{0} \kappa \beta_{\perp}^{3 / 5}}\left(\frac{25 d_{0} e^{2} n_{0}^{2}}{8}\right)^{2 / 5} \frac{1-r_{d}}{\left(1-r_{d}^{5 / 2}\right)^{2 / 5}},
$$

where we have defined the dimensionless parameter $r_{d}=n^{2 / 3}(d) / n^{2 / 3}(0)$. Although these analyses were for the zero temperature limit, we do not expect relevant finite temperature effects at these carrier densities [32].

The potential difference obtained from the above model is shown, for different carrier concentrations, in Fig. 4(a) for a $n$-doped sample (see the Supplemental Material for results also on $p$-doped samples [22]). The screening of charged impurities or the gate potential increases as the thickness of the BP layer grows. The dependence of $\Delta V(d)$ on $d$ suggests an intermediate screening behavior between the strong coupling limit of graphene, where the carriers concentrate close to the interface [31] and the weak coupling regime with reduced screening properties that dominates the screening of $\mathrm{MoS}_{2}$ [33]. Our results suggest that the gate will have negligible effect, $10 \mathrm{~nm}$, into the bulk of BP, consistent with recent experiments on multilayer BP transistors [9]. We have also calculated $n(z)$ for a sample with a given thickness $d$ but different charge carrier concentrations $n_{0}$ as shown in Fig. 4(b). We observe a strong dependence of the screening strength on $n_{0}$. We infer a screening length of the order of the interlayer spacing for $\sigma_{0}=10^{13} \mathrm{~cm}^{-2}$, whereas for lower 
concentrations, like $10^{11} \mathrm{~cm}^{-2}$, the screening length is an order of magnitude larger.

Conclusions. - In conclusion, whereas we find a relatively isotropic static screening, the band nonparabolicity leads to highly anisotropic plasmons, whose resonance scales with doping as $n^{\beta}$, where $\beta<\frac{1}{2}$. Furthermore, the modes dispersing along one of the crystallographic directions are long lived, being Landau damped only for near infrared frequencies. Finally, we find that the strength of the electric field screening in BP flakes seem to be between the strong coupling regime characteristic of graphene, and the weak coupling regime of the TMD semiconductors, such as $\mathrm{MoS}_{2}$.

F. G. and R. R. acknowledge support from the Spanish Ministry of Economy (MINECO) through Grant No. FIS2011-23713, the European Research Council Advanced Grant (Contract No. 290846), and the European Commission under the Graphene Flagship, Contract No. CNECT-ICT-604391. R. R. acknowledges financial support from the Juan de la Cierva Program.

*tonyaslow@gmail.com

[1] R. W. Keyes, Phys. Rev. 92, 580 (1953).

[2] D. Warschauer, J. Appl. Phys. 34, 1853 (1963).

[3] J. C. Jamieson, Science 139, 1291 (1963).

[4] A. Morita, Appl. Phys. A 39, 227 (1986).

[5] K. J. Chang and M. L. Cohen, Phys. Rev. B 33, 6177 (1986).

[6] E. S. Reich, Nature (London) 506, 19 (2014).

[7] L. Li, Y. Yu, G. J. Ye, Q. Ge, X. Ou, H. Wu, D. Feng, X. H. Chen, and Y. Zhang, Nat. Nanotechnol. 9, 372 (2014).

[8] H. Liu, A. T. Neal, Z. Zhu, D. Tomanek, and P. D. Ye, ACS Nano 8, 4033 (2014).

[9] F. Xia, H. Wang, and Y. Jia, Nat. Commun. 5, 4458 (2014).

[10] S. P. Koenig, R. A. Doganov, H. Schmidt, A. H. Neto, and B. Oezyilmaz, Appl. Phys. Lett. 104, 103106 (2014).

[11] A. Castellanos-Gomez, L. Vicarelli, E. Prada, J. O. Island, K. L. Narasimha-Acharya, S. I. Blanter, D. J. Groenendijk et al., 2D Materials 1, 025001 (2014).

[12] T. Low, A. S. Rodin, A. Carvalho, Y. Jiang, H. Wang, F. Xia, and A. H. C. Neto, arXiv:1404.4030 [Phys. Rev. B (to be published)].
[13] T. Low, M. Engel, M. Steiner, and P. Avouris, arXiv: 1407.7286 [Phys. Rev. B (to be published)].

[14] A. S. Rodin, A. Carvalho, and A. H. Neto, Phys. Rev. Lett. 112, 176801 (2014).

[15] V. Tran, R. Soklaski, Y. Liang, and L. Yang, Phys. Rev. B 89, 235319 (2014).

[16] A. N. Rudenko and M. I. Katsnelson, Phys. Rev. B 89, 201408 (2014).

[17] S. ichiro Narita, S. ichi Terada, S. Mori, K. Muro, Y. Akahama, and S. Endo, J. Phys. Soc. Jpn. 52, 3544 (1983).

[18] L. F. Mattheiss, Phys. Rev. B 8, 3719 (1973).

[19] J. Lindhard, Kgl. Danske Videnskab. Selskab Mat.-Fys. Medd. 28, 8 (1954).

[20] C. Kittel, Introduction to Solid State Physics (Wiley, New York, 2004).

[21] G. F. Giuliani and G. Vignale, Quantum Theory of the Electron Liquid (Cambridge University Press, Cambridge, 2005).

[22] See Supplemental Material at http://link.aps.org/ supplemental/10.1103/PhysRevLett.113.106802 for the results on dynamic screening, interlayer screening for $p$-doped case, and experimental data on multilayer BP.

[23] A. N. Grigorenko, M. Polini, and K. S. Novoselov, Nat. Photonics 6, 749 (2012).

[24] T. Low and P. Avouris, ACS Nano 8, 1086 (2014).

[25] T. Stauber, J. Phys. Condens. Matter 26, 123201 (2014).

[26] H. Yan, T. Low, W. Zhu, Y. Wu, M. Freitag, X. Li, F. Guinea, P. Avouris, and F. Xia, Nat. Photonics 7, 394 (2013).

[27] L. Ju, B. Geng, J. Horng, C. Girit, M. Martin, Z. Hao, H. A. Bechtel et al., Nat. Nanotechnol. 6, 630 (2011).

[28] Z. Fei, A. S. Rodin, G. O. Andreev, W. Bao, A. S. McLeod, M. Wagner, L. M. Zhang et al., Nature (London) 487, 82 (2012).

[29] J. Chen, M. Badioli, P. Alonso-Gonzlez, S. Thongrattanasiri, F. Huth, J. Osmond, M. Spasenović et al., Nature (London) 487, 77 (2012).

[30] L. Pietronero, S. Strässler, H. R. Zeller, and M. J. Rice, Phys. Rev. Lett. 41, 763 (1978).

[31] S. S. Datta, D. R. Strachan, E. J. Mele, and A. C. Johnson, Nano Lett. 9, 7 (2009).

[32] M. A. Kuroda, J. Tersoff, and G. J. Martyna, Phys. Rev. Lett. 106, 116804 (2011).

[33] A. C. Gomez, E. Cappelluti, R. Roldn, N. Agrat, F. Guinea, and G. Rubio-Bollinger, Adv. Mater. 25, 899 (2013). 